\title{
A hybrid Gerchberg-Saxton-like algorithm for DOE and CGH calculation
}

\author{
Haichao Wang ${ }^{1,2}$, Weirui Yue ${ }^{1,2}$, Qiang Song ${ }^{1,2}$, Jingdan Liu ${ }^{1,3}$, and \\ Guohai Situ ${ }^{1, *}$ \\ 1 Shanghai Institute of Optics and Fine Mechanics, Chinese Academy of Sciences, Shanghai \\ 201800, China \\ 2 University of the Chinese Academy of Sciences \\ 3 School of Optoelectronics, Beijing Institute of Technology, Beijing 100081, China \\ * Email address: ghsitu@siom.ac.cn
}

\begin{abstract}
The Gerchberg-Saxton (GS) algorithm is a widely used in various disciplines of modern sciences and technologies where phase retrieval is required. However, this legendary algorithm most likely stagnates after a few iterations. Many efforts have been taken to improve this situation. Here we propose to introduce the strategy of gradient descent and weighting technique to the GS algorithm, and demonstrate it using two examples: design of a diffractive optical element (DOE) to achieve off-axis illumination in lithographic tools, and design of a computer generated hologram (CGH) for holographic display. Both numerical simulation and optical experiments are carried out for demonstration.
\end{abstract}

Keywords: Phase Retrieval, Gerchberg-Saxton Algorithm, Holographic Display, Diffractive Optical Element, Laser Beam Shaping.

\section{Introduction}

The Gerchberg-Saxton (GS) algorithm [1] is one of the most popular algorithms used for phase retrieval, which is a key mathematical model for many problems such as the calculation of computer-generated holograms (CGHs) [2, $3,4]$, the design of diffractive optical elements (DOE) $[5,6]$, the restoration of image from the magnitude of its Fourier spectrum $[7,8]$, and coherent diffractive imaging in both the optical [9] and X-ray [10] regime. GS-based routines have also been used for optical image encryption [11, 12] and image hiding [13] in a cascaded architecture, resolution enhancement for wide-field microscopy [14], and ghost imaging [15].

To put forward, let us start from the very original problem of phase retrieval. It is now well-known that the problem can be mathematically stated as $[9$, 10, 16]: given the intensity measurement of a scattering or diffraction field, $I(\boldsymbol{p})=|F(\boldsymbol{p})|^{2}$ and some a priori knowledge about the object $f(\boldsymbol{r})$, retrieve the 
missing phase, $\exp [j \psi(\boldsymbol{p})]$ of the diffraction field, so that the following relation is valid

$$
\begin{aligned}
|F(\boldsymbol{p})| \exp [j \psi(\boldsymbol{p})] & =\mathscr{F}\{f(\boldsymbol{r})\} \\
& =\iint_{-\infty}^{\infty} f(\boldsymbol{r}) \exp [-j 2 \pi \boldsymbol{r} \cdot \boldsymbol{p}] \mathrm{d}^{2} \boldsymbol{r},
\end{aligned}
$$

where $\boldsymbol{r}=(x, y)$ and $\boldsymbol{p}=(u, v)$ are the coordinates of the real (or object) space and the Fourier (or recording, or output) space, respectively, and the operator $\mathscr{F}$ represents the Fourier transform. Nowadays, the intensity $I(\boldsymbol{p})$ is usually captured using digital cameras such as CCDs or CMOSs, the data is discrete and the Fourier transform in Eq. (1) is usually represented by the discrete Fourier transform (DFT). Equation (1) models the physical systems that $F(\boldsymbol{p})$ is the Fourier transform of $f(\boldsymbol{r})$. This includes systems of X-ray diffraction and the shaping of the illumination beam in the lithographic tool. However, one should bear in mind that this model is not limited to such systems. The Fourier transform can be generalized to any linear canonical transform such as the Fresnel and the fractional Fourier transform [17, 18].

From a mathematical perspective, Eq. (1) is equivalent to be the following optimization problem [7]

$$
\min \||F(\boldsymbol{p})|-|\mathscr{F}\{|f(\boldsymbol{r})| \exp [j \phi(\boldsymbol{r})]\}|\|
$$

for the missing phase $\phi(\boldsymbol{r})$, where $\|\cdot\|$ is the norm. Gerchberg and Saxton [1] were the first researchers who give a practical solution to this problem now beard their name.

Numerically, the GS algorithm contains the forward and inverse Fourier (or, generally speaking, canonical) transforms between the real (object) space and the Fourier (image) space, in which the measured modulus are applied to the calculated wavefront. Fienup has shown that the GS algorithm "converges" in the weak sense, i.e., the square error cannot increase with the increasing number of iterations [16], leading to a algorithmic phenomenon called stagnation $[19,20]$, which prohibits the searching of the solution to the problem. In the past decades, various modifications have been proposed to improve the GS algorithm. For example, Fienup's group of Input-Output algorithms [16] have been proposed to improve the stagnation; Yang-Gu algorithm [21] has been proposed accounting for non-unitary transforms; the weighting strategy [22, 23] have been proposed to improve the design of diffractive elements; and Wyrowski and Bryngdahl [5] have proposed a method to synthesize a better initial guess. However, all these methods have pros and cons. For example, Fienup's algorithms have a big convergence issue [24] and are time- consuming in numerical implementation; Yang-Gu's algorithm does not show significant improvement as holographic display is usually a unitary system; and the weighting strategy usually defines a series of intermediate images of the object to be encoded, and that decreases the computational efficiency as well. Here in this manuscript, we propose a hybrid algorithm by introducing two convergence-accelerating strategy into the GS routine: the gradient descent to the phase is employed in every 
iteration at the real space, and the weighted strategy is called at the Fourier space when a local minimum is found. We will show that the number of iterations the proposed algorithm needs to run is not more than the GS algorithm, while better solution can be found.

In the next section, we first provide a brief review of the GS algorithm, and then describe the proposed algorithm in detail. The performance of the proposed algorithm is demonstrated in Section 3 with the proof-of-principle experiments of laser beam shaping for lithographic illumination system and holographic display.

\section{Algorithms}

\subsection{The Gerchberg-Saxton algorithm}

Mathematically, the GS routine to solve Eq. (2) can be written as the iterative projection onto constraint (nonconvex) sets in a Hilbert space [7, 24]

$$
f_{k+1}=\mathcal{P}_{1} \mathcal{P}_{2} f_{k}
$$

where $\mathcal{P}_{1}$ is the projection operator onto the constraint set in the real space, $\mathcal{P}_{2}$ is the projection operator onto the constraint set in the Fourier space, and $f_{k}$ is the image retrieved at the $k^{\text {th }}$ iteration. For all closed sets, the projection $g \equiv \mathcal{P} f$ of $f$ onto the set $\mathrm{C}$ can be defined as

$$
\|g-f\|=\min \|y-f\|, \quad \forall g, y \in \mathrm{C} .
$$

One significant difference between different phase retrieval algorithms is the way to define the constraint sets. In the GS routine, the set in the real plane is defined as

$$
\mathrm{C}_{1}=\{g(\boldsymbol{r}): \quad g(\boldsymbol{r})=0, \quad \forall|\boldsymbol{r}| \notin \boldsymbol{a}\}
$$

if the finite support (defined by $\boldsymbol{a}$ ) constraint is applied. That is, replacing any value outside the signal window of the iterative amplitude with 0 . Similarly, the set in the Fourier plane is usually defined as

$$
\mathrm{C}_{2}=\{g(\boldsymbol{r}) \leftrightarrow G(\boldsymbol{p}): \quad|G(\boldsymbol{r})|=F(\boldsymbol{p}), \quad \forall \boldsymbol{p}\}
$$

where $F$ is the measured magnitude. It is easy to verify that $C_{1}$ is convex and $\mathrm{C}_{2}$ is not. The projection operators $\mathcal{P}_{1}$ and $\mathcal{P}_{2}$ are then given by

$$
\mathcal{P}_{1} g(\boldsymbol{r})= \begin{cases}g(\boldsymbol{r}), & |\boldsymbol{r}| \in \boldsymbol{a} \\ 0, & |\boldsymbol{r}| \notin \boldsymbol{a}\end{cases}
$$

and

$$
\mathcal{P}_{2} g(\boldsymbol{r}) \leftrightarrow F(\boldsymbol{p}) \exp [j \varphi(\boldsymbol{p})]
$$

where $\varphi(\boldsymbol{p})$ is the phase of $G(\boldsymbol{p})$. This means that the magnitude is replaced by the measured data, while the phase is unchanged in the Fourier domain.

In the cases of DOE design and CGH calculations, the input beam $f(\boldsymbol{r})$ is usually known (to be a plane wave, for instance), or can be calibrated. The 
phase-retrieval problem can be stated as to find a phase function $\exp [j \phi(\boldsymbol{r})]$ so that the Fourier (or the linear canonical) transform of $f(\boldsymbol{r}) \exp [j \phi(\boldsymbol{r})]$ satisfies some predefined criteria. This problem can be solved using the iteration process described by Eq. (3) as well, provided that the constraint set in the real space is written as

$$
\mathrm{C}_{1}=\{f(\boldsymbol{r}): \quad g(\boldsymbol{r})=0, \quad \forall|\boldsymbol{r}| \notin \boldsymbol{a}\} .
$$

This means that the amplitude in the real space is replaced by $f(\boldsymbol{r})$ within the signal window, and forced to be 0 otherwise.

As suggested by Eq. (3), one obvious property of the GS algorithm is that the solution is strongly dependent on the initial condition $f_{0}$, very probably leading to stagnation [19]. Many efforts have been taken to avoid such an algorithmic phenomenon. Fox example, Wyrowski and Bryngdahl [5] have proposed to synthesize an iterative phase after a few initial guesses, Fienup has proposed the hybrid input-output algorithms [16], and Zhang et al. [22] and Kuzmenko [23] has proposed the weighted strategy.

\subsection{The proposed algorithm}

With a close examination of the above modified GS-like algorithms, one can conclude that they fall into two main categories. The first one is to the modify the iterative amplitude in the real space by introducing freedom outside the signal window [5], or by introducing a feedback mechanics [16], or by modifying the input function $f(\boldsymbol{r})$ by a weighted factor [23]. The second one is to modify the target image $|F(\boldsymbol{p})|$ by a weighted coefficient [22].

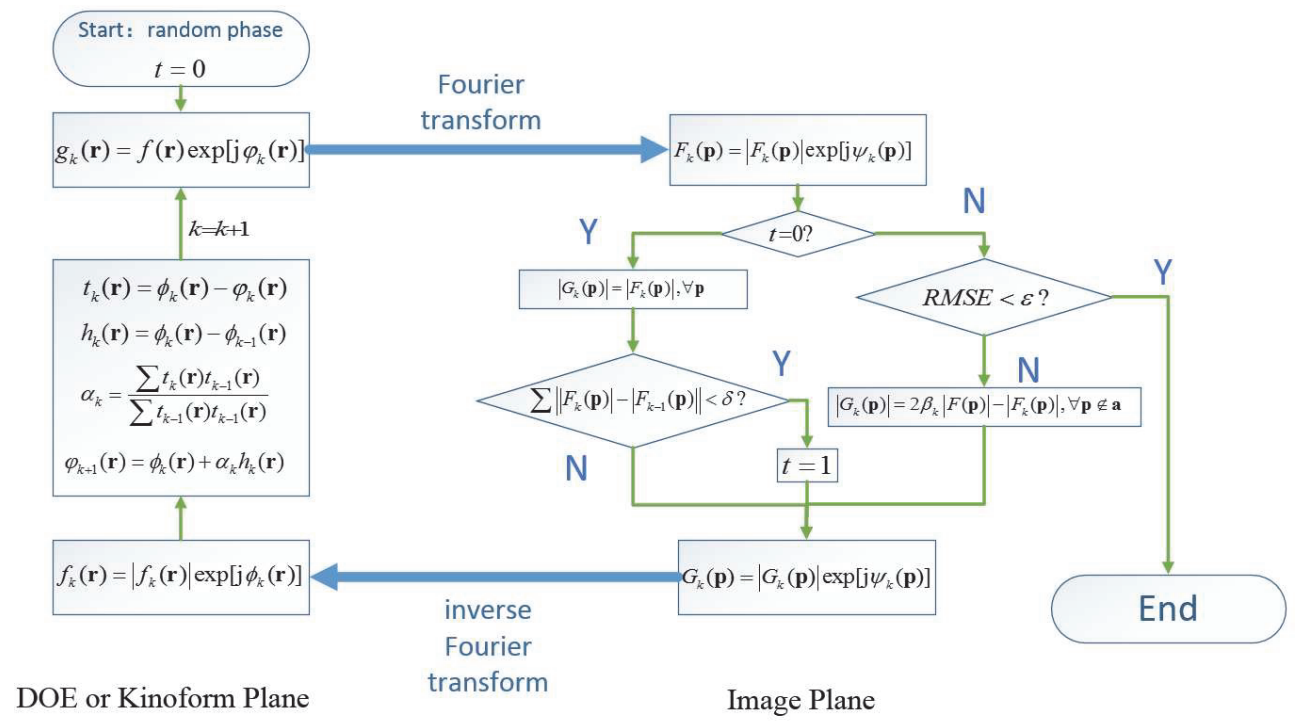

Figure 1: Block diagram of the proposed phase retrieval algorithm. 
In this subsection, we propose an alternative algorithm. Unlike the methods mentioned above, the proposed algorithm manages to introduce the phase gradient descent in the real space and the modification of the constraint in the Fourier space, as shown in the block diagram in Fig. 1. The detailed description of the algorithm is as following. In each iteration of the standard GS routine, the wavefront computed in the real space $\left|f_{k}(\boldsymbol{r})\right|$ is constrained by the a priori knowledge. Specifically, in the real space, besides the replacement of $\left|f_{k}(\boldsymbol{r})\right|$ with $|f(\boldsymbol{r})|$, as in the standard GS algorithm, a strategy of gradient descent is introduced to modify the phase component as well. This is done by modulating the phase $\varphi_{k}(\boldsymbol{r})$ (of the DOE or CGH) obtained in the $k^{\text {th }}$ iteration as

$$
\varphi_{k+1}(\boldsymbol{r})=\phi_{k}(\boldsymbol{r})+\alpha_{k} h_{k}(\boldsymbol{r}),
$$

where $\left|h_{k}(\boldsymbol{r})\right|$ is direction of gradient defined as $\nabla f_{k}(\boldsymbol{r})$, which is actually proportional to the difference between the phase obtained at the current iteration and the most recent iteration

$$
h_{k}=\phi_{k}(\boldsymbol{r})-\phi_{k-1}(\boldsymbol{r})
$$

and $\alpha_{k}$ is an acceleration coefficient defined as

$$
\alpha_{k}=\frac{\Sigma t_{k}(\boldsymbol{r}) t_{k-1}(\boldsymbol{r})}{\sum t_{k-1}(\boldsymbol{r}) t_{k-1}(\boldsymbol{r})},
$$

where

$$
t_{k}(\boldsymbol{r})=\phi_{k}(\boldsymbol{r})-\varphi_{k}(\boldsymbol{r}) .
$$

Then the resulting phase $\varphi_{k+1}(\boldsymbol{r})$ is multiplied with the input profile $f(\boldsymbol{r})$ and form the complex amplitude $g_{k}(\boldsymbol{r})=f(\boldsymbol{r}) \exp \left[j \varphi_{k+1}(\boldsymbol{r})\right]$ for the next iteration.

One should take more careful in the Fourier space. At the beginning, a standard constraint set can be used,i.e., the constraint set defined in the way similar to Eq. (6)

$$
\mathrm{C}_{2}=\{g(\boldsymbol{r}) \leftrightarrow G(\boldsymbol{p}): \quad|G(\boldsymbol{r})|=|F(\boldsymbol{p})|, \quad \forall \boldsymbol{p}\}
$$

However, the constraint set defined in this way alone only drives the error to drop steeply within a few iterations at the beginning; and the error keeps decreasing very slowly afterwards as the GS algorithm, although phase gradient has been applied to the real space. To make a further drop, here we propose to introduce a weighted algorithm when the decreasing of the error becomes insignificant, that is, the RMSE value between the two calculated patterns obtained at two contiguous iterations with a predefined threshold $\delta$, i.e.,

$$
\sum|| F_{k}(\boldsymbol{p})|-| F_{k-1}(\boldsymbol{p})||<\delta \text {. }
$$

When this condition is satisfied, an alternative constraint set defined as

$$
\mathrm{C}_{2}^{\prime}=\left\{g(\boldsymbol{r}) \leftrightarrow G(\boldsymbol{p}): \quad|G(\boldsymbol{r})|=2 \beta_{k}|F(\boldsymbol{p})|-\left|F_{k}(\boldsymbol{p})\right|, \quad \forall \boldsymbol{p} \in \boldsymbol{a}\right\}
$$


where $\boldsymbol{a}$ is the signal window, and the weighted factor $\beta_{k}$ is

$$
\beta_{k}=\frac{\sum\left|F_{k}(\boldsymbol{p})\right|}{\sum|F(\boldsymbol{p})|}, \quad \forall \boldsymbol{p} \in \boldsymbol{a} .
$$

In the applications such as DOE and CGH design, one usually uses the rootmean-square-error (RMSE) as the merit of convergence. The RMSE is defined as

$$
\mathrm{RMSE}=\left[\frac{\sum\left(\left|F_{k}\right|-F\right)^{2}}{\sum F^{2}}\right]^{\frac{1}{2}} .
$$

The algorithm is ended when RMSE reaches the predefined criterion threshold $\epsilon$.

\section{Applications}

In this section, we provide numerical and experimental demonstration of the proposed algorithm with two examples: the design of DOE for laser beam shaping for the application in lithographic tools, and the design of CGH for display.

\subsection{Design of DOE for off-axis illumination in lithographic tool}

Off axis illumination is one of the key techniques for resolution enhancement in projection lithography system [25]. This is implemented by the phase-only DOEs in the pupil shaping unit in most of the deep-ultraviolet lithography machines owing to their design flexibility, high-quality freeform illumination mode generation and stable output against the fluctuation of the input laser beam. There are several key factors that affect the exposure performance of the pupil shaping unit such as opening angle, azimuth angle, and efficiency. Among these factors, high efficiency is mostly desirable as this means shorter exposure time, leading directly to the increase of lithography throughput [26]. As an example, we design a DOE to implement annular illumination with the ratio $\sigma_{\text {inner }}: \sigma_{\text {outer }}=1: 2$ using the proposed algorithm. Here $\sigma_{\text {inner }}$ and $\sigma_{\text {outer }}$ stand for the inner and outer radius of the annular illumination, respectively, as shown in Fig. 2(a).

The design result is shown in Fig. 2(b). From this, one has an impression that the difference between the designed output pattern and the target pattern is negligible. This is more clearly seen when examining their cross sections, which are shown in Fig. 2(d) and (e), respectively. The RMSE value between the obtained pattern and the target is 0.0194. For comparison, we ran the GS algorithm with the same initial condition, and ended up with the output diffraction pattern shown in Fig. 2(c). It is clearly that noise appears in the annulus area, as can be perceptually seen from the cross section plotted in Fig. 2(f). The RMSE value between Fig. 2(c) and Fig. 2(a) is just 0.0875. 

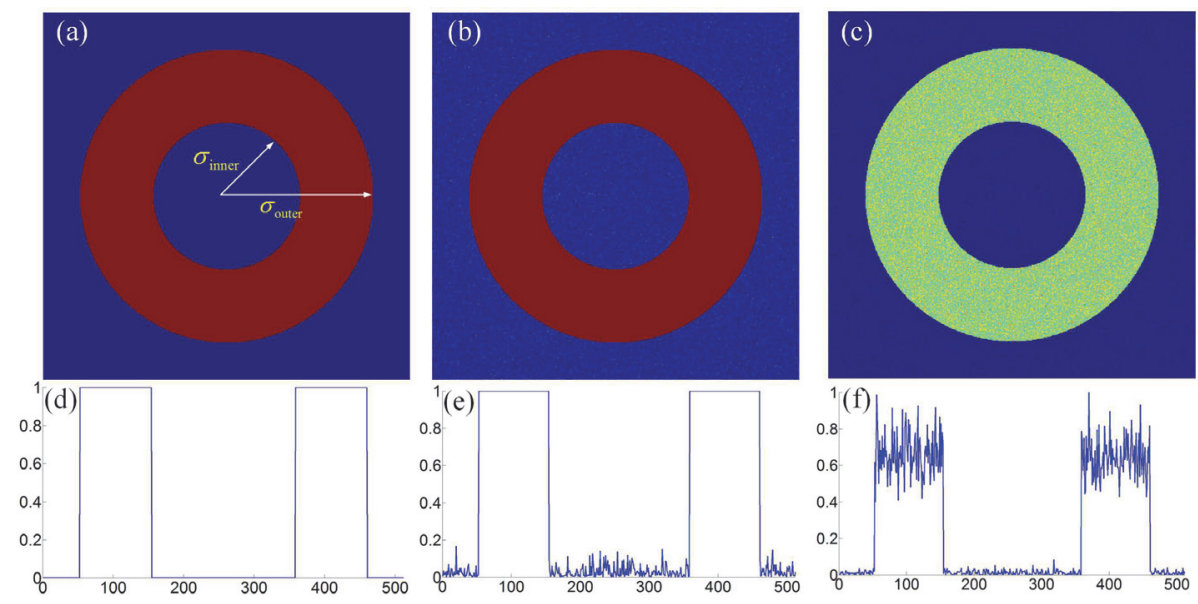

Figure 2: Simulation results of DOE design for off-axis illumination. (a) Intensity distribution of target uniform annular illumination, and design result using the (b) proposed algorithm and (c) GS algorithm. (d), (e) and (f) are cross section intensity distribution of (a), (b) and (c), respectively.

For off-axis illumination, one more important criterion is the energy variation within the annulus. This is usually evaluated by using a parameter called imbalance, which is defined as [26]

$$
B=\frac{I_{\max }-I_{\min }}{I_{\max }+I_{\min }} .
$$

The imbalance value of the annulus pattern obtained by using the GS algorithm is 0.6521 in our design. However, this value is as small as $5.268 \times 10^{-4}$ for the proposed algorithm, 3 order of magnitude smaller than that of the GS algorithm. This indicates that the DOE designed by the proposed algorithm can produce a much flatter annulus pattern with respect to the GS algorithm, as clearly shown in Fig. 2(e) and (f).

Further examination reveals that the diffraction efficiency of Fig. 2(c) within the annulus is 0.9711 , in comparison to 0.9519 of Fig. 2(b). This indicates that the diffraction efficiency of the DOE designed by the proposed algorithm is a slightly less than that obtained by the GS algorithm. This can be clearly seen that the noise level outside the signal window in Fig. 2(e) is a slightly higher than that in Fig. 2(f). The reason is that the proposed algorithm introduces freedom outside the signal window in the output plane, as suggested by Eq. (16), whereas the GS algorithm imposes a hard constraint there.

To examine the convergence behavior of the proposed algorithm, we plot the RMSE value between the calculated and the target patterns in the logarithmic scale versus the iteration number in Fig. 3. Each data point was obtain by averaging 20 runs with different initial conditions. The first thing Fig. 3 suggests is that the deviation of these points is very small, indicating that the initial guess has little effect to the convergence, although the resulting intensity distribution 


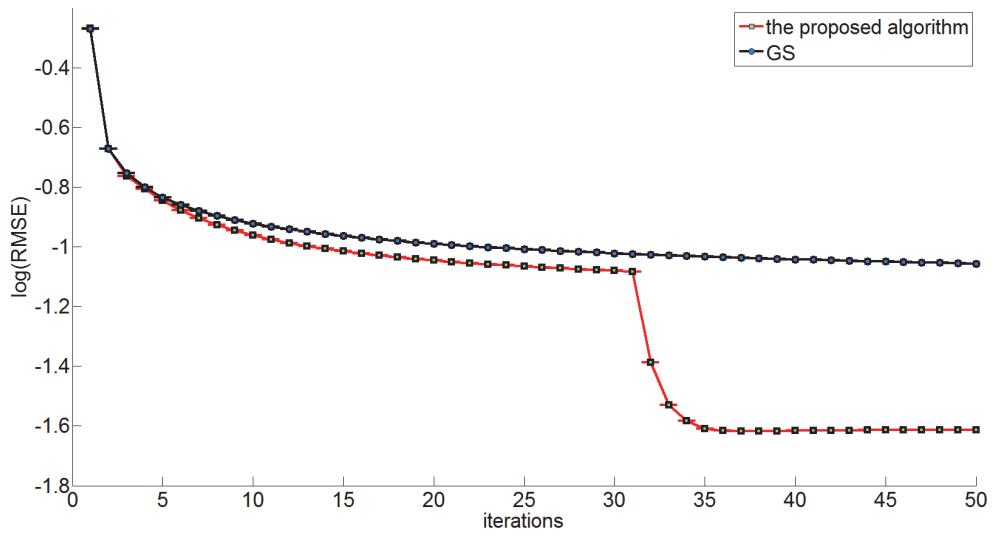

Figure 3: Convergence behavior of the proposed algorithm and the GS algorithm.

may be slightly different. The result also suggests that the proposed algorithm converges faster than the GS algorithm from the very beginning, mainly owing to the introduction of the gradient descent in the DOE plane. One can understand this with the help of Fig. 4, which illustrates the strategies of solution-searching of these two algorithms. Unlike the step-by-step jumping from the current guess along the projection direction to the next best guess in the GS algorithm, the proposed algorithm attempts to search the next best guess along the phase gradient descent direction as suggested in Eq. (10), thus leading to a bigger step of jumping, and faster convergence. Although the use of gradient descent alone ensures faster convergence, the error curve becomes flat after a few iterations as the GS algorithm, indicating the signature of stagnation. When this occurs, the

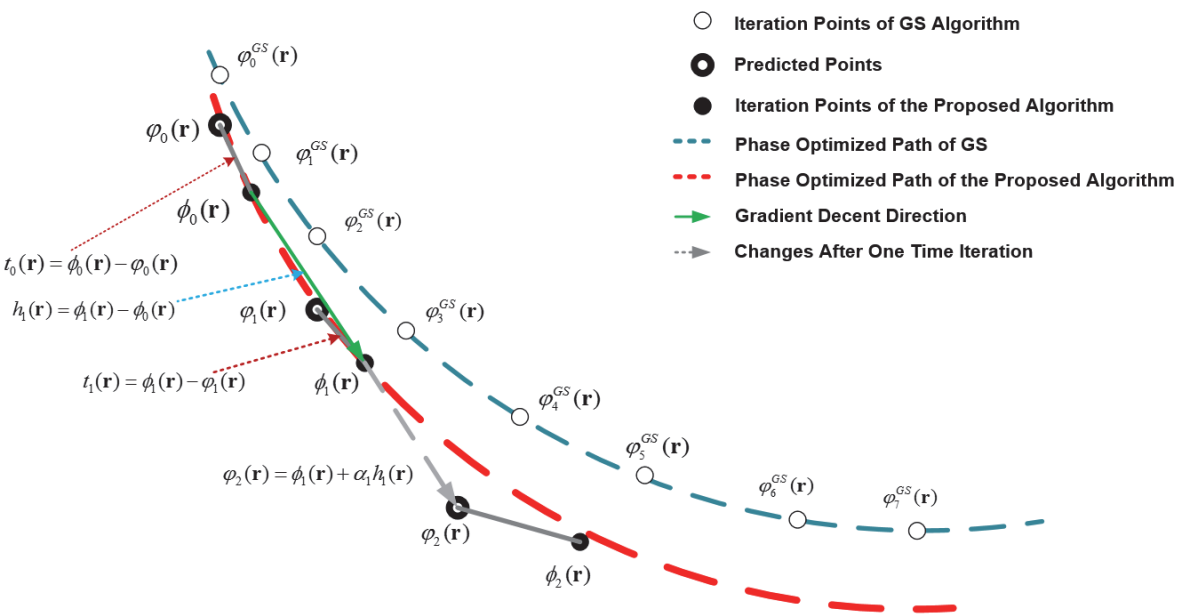

Figure 4: The convergence principle of descent gradient 

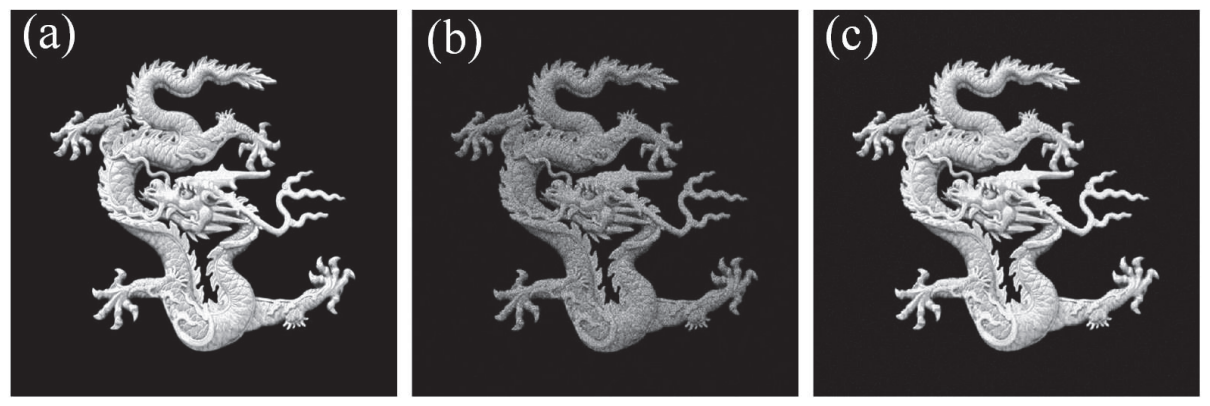

Figure 5: Simulation results of CGH design for holographic display. (a) the target image, and the numerically reconstructed image from the CGH designed using (b) the GS and (c) the proposed algorithm.

proposed algorithm invokes the weighting strategy within the signal window and introduces freedom outside at the output plane. The convergence curve plotted in Fig. 3 clearly shows a second steep drop of the RMSE value when it happens.

\subsection{Design of CGH for holographic display}

The second example to demonstrate the proposed algorithm is to design a $\mathrm{CGH}$ for holographic display. The target image is a Chinese dragon as shown in Fig. 5(a). The simulation results plotted in Fig. 5(b) and (c) are the reconstructed images from the CGH designed using the GS and the proposed algorithm, respectively. Perceptually, we can clearly see that the results obtained by the proposed algorithm is far better than the one reconstructed by the GS algorith$\mathrm{m}$. Numerical calculations show that the RMSE value associated with these two reconstructed images are 0.1271 and 0.046 , respectively.

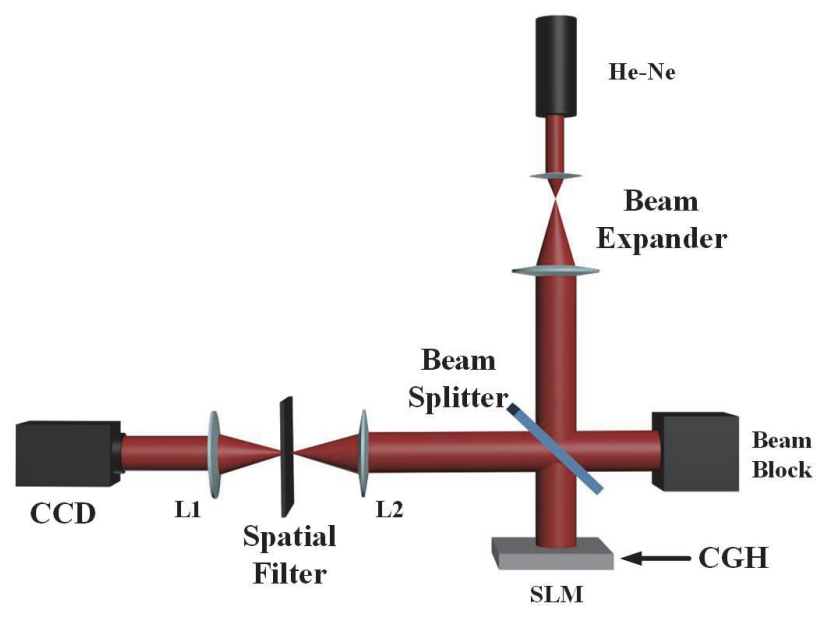

Figure 6: Schematic optical setup for holographic display. 
We also carried out optical experiment to demonstrate the algorithm. The optical setup of our holographic display system is schematically shown in Fig. 6. The laser beam emitted from a stabilized He-Ne laser (Thorlabs, wavelength $633 \mathrm{~nm}$, output power $1.2 \mathrm{~mW}, \mathrm{TEM}_{00}$ mode, linear polarized) was first expanded and collimated and then directed to illuminate a Liquid Crystal on Silicon (LCoS) spatial light modulator(Holoeye, LETO). The LCoS panel has $1920 \times 1080$ pixels, each of which is $6.4 \mu \mathrm{m} \times 6.4 \mu \mathrm{m}$ in size. With proper gammacurve correction, this LCoS model can offer 8-bits phase modulation within $[0,2 \pi]$ at $633 \mathrm{~nm}$ wavelength. Thus, the CGH designed using either algorithm should be quantized to 8-step phase levels in order to be correctly displayed onto the LCoS. The quantization can be usually done either in each algorithmic iteration, or when the final solution is found. In our demonstration, we employed the second method for simplicity. The reconstruction from the CGH was obtained by the Fourier lens L1 when the LCoS was illuminated by the collimated laser beam. The zeroth-order diffraction appears at the center of the Fourier plane; and its intensity is very strong as expected. So we used a spatial filter to select the first-order diffraction, which reveals the optical reconstructed image. In our experiment, we captured the reconstructed image by a CCD camera with an imaging lens L2. The results are shown in Fig. 7. It is apparent that the experimental reconstruction results are consistent with the simulation, as one can see that the eyes and scales of the dragon are more clearly reconstructed using the CGH designed by our algorithm [highlighted in Fig. 7(b)], and the overall image is sharper, although we did not take any effort to reduce the laser speckles.

We show in Fig. 8 another example: the optical reconstruction of the image
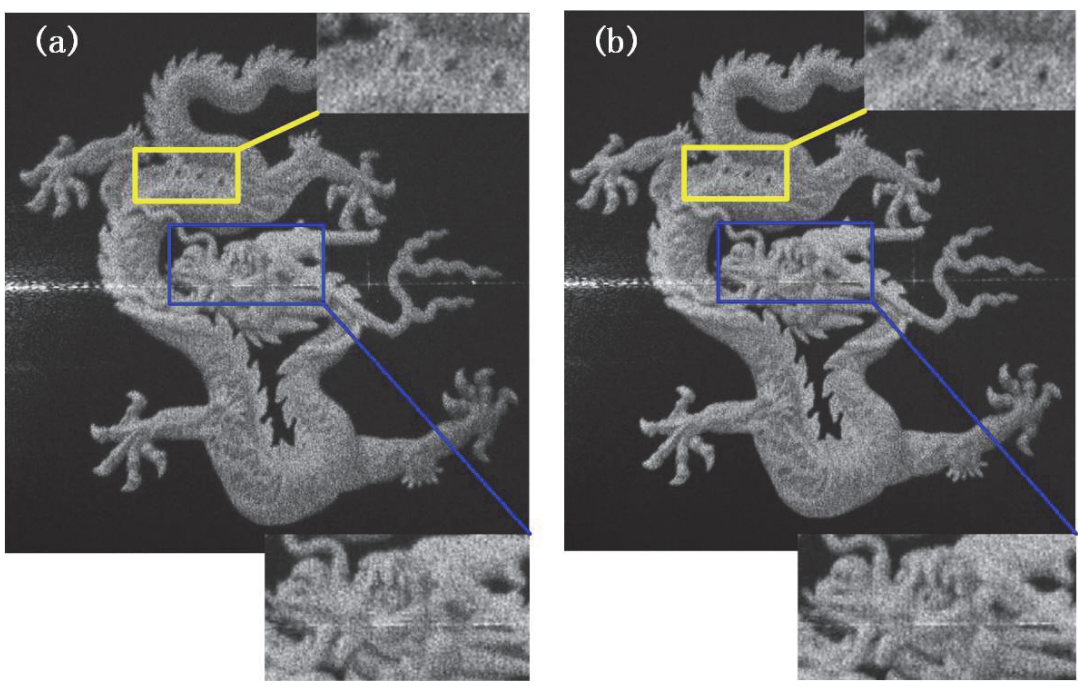

Figure 7: Experimental results: optical reconstructions of the Chinese dragon from the CGH calculated by using (a) the GS and (b) the proposed algorithm. 

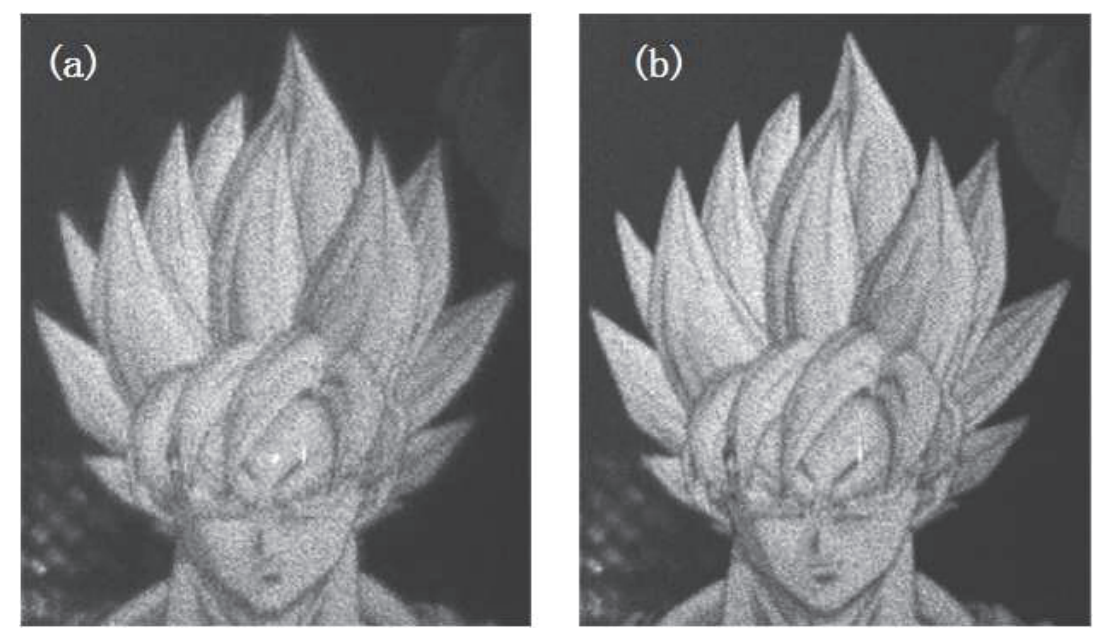

Figure 8: Experimental results: optical reconstructions of the image Goku from the CGH calculated by using (a) the GS and (b) the proposed algorithm.

Goku, which is a typical cartoon character. It is even clearer in this case that the image reconstructed from the CGH calculated using the proposed method is much better in terms of contrast and sharpness than the other one.

\section{Conclusion}

In conclusion, we have generalized the GS algorithm by introducing the strategy of gradient descent in real space, and a weighting mechanics in the Fourier plane. Simulation and optical experiments of laser beam shaping and holographic display have been carried out to demonstrate that the overall performance of the proposed method is better than the GS algorithm. Furthermore, the proposed algorithm can be used for various other applications such as phase imaging, and optical image encryption.

\section{Acknowledgements}

This study was supported by the National Science Foundation of China (Grants 61377005, 61327902), the Recruitment Program of Global Youth Experts, and the Shanghai Pujiang Program.

\section{Reference}

[1] R. W. Gerchberg, W. O. Saxton, A practical algorithm for the determination of phase from image and diffraction plane pictures, Optik 35 (1972) 237-246. 
[2] G. Zheng, H. Mühlenbernd, M. Kenney, G. Li, T. Zentgraf, S. Zhang, Metasurface holograms reaching 80\% efficiency, Nat. Nanotechnol. 10 (2015) 308-312.

[3] L. Huang, X. Chen, H. Mühlenbernd, H. Zhang, S. Chen, B. Bai, Q. Tan, G. Jin, K.-W. Cheah, C.-W. Qiu, J. Li, T. Zentgraf, S. Zhang, Threedimensional optical holography using a plasmonic metasurface, Nat. Commun. 4 (2013) 2808.

[4] E. Buckley, Hologrphic laser projection, J. Disp. Tech. 7 (2011) 135-140.

[5] F. Wyrowski, O. Bryngdahl, Iterative Fourier-transform algorithm applied to computer holography, J. Opt. Soc. Am. A 5 (1988) 1058-1065.

[6] J. Liu, M. Taghizadeh, Iterative algorithm for the design of diffractive phase elements for laser beam shaping, Optics Letters 27 (2002) 1463-1465Ge.

[7] A. Levi, H. Stark, Image restoration by the method of generalized projections with application to restoration from magnitude, J. Opt. Soc. Am. A 1 (1984) 932-943.

[8] D. S. Biggs, M. Andrews, Acceleration of iterative image restoration algorithms, Appl. Opt. 36 (1997) 1766-1775.

[9] Y. Shechtman, Y. C. Eldar, O. Cohen, H. N. Chapman, J. Miao, M. Segev, Phase retrieval with application to optical imaging: A contemporary overview, IEEE Signal Processing Mag. 32 (2015) 87-109.

[10] J. Miao, T. Ishikawa, I. K. Robinson, M. M. Murnane, Beyond crystallography: Diffractive imaging using coherent x-ray light sources, Science 348 (2015) 530-535.

[11] G. Situ, J. Zhang, A lensless optical security system based on computergenerated phase only masks, Opt. Commun. 232 (2004) 115-122.

[12] W. Chen, G. Situ, X. Chen, High-flexibility optical encryption via aperture movement, Opt. Express 21 (2013) 24680-24691.

[13] Y. Shi, G. Situ, J. Zhang, Multiple-image hiding in the Fresnel domain, Opt. Lett. 32 (2007) 1914-1916.

[14] G. Zheng, R. Horstmeyer, C. Yang, Wide-field, high-resolution Fourier ptychographic microscopy, Nat. Photon. 7 (2013) 739-746.

[15] W. Wang, X. Hu, J. Liu, S. Zhang, J. Suo, G. Situ, Gerchberg-Saxton-like ghost imaging, Opt. Express 23 (2015) 28416-28422.

[16] J. R. Fienup, Phase retrieval algorithms: a comparison, Appl. Opt. 21 (1982) 2758-2769. 
[17] Z. Zalevsky, R. G. Dorsch, Gerchberg-Saxton algorithm applied in the fractional fourier or the fresnel domain, Opt. Lett. 21 (1996) 842-844.

[18] C. Guo, S. Liu, J. T. Sheridan, Iterative phase retrieval algorithms. I: optimization, Appl. Opt. 54 (2015) 4698-4708.

[19] J. R. Fienup, C. C. Wackerman, Phase-retrieval stagnation problems and solutions, J. Opt. Soc. Am. A 3 (1986) 1897-1907.

[20] H. Takajo, T. Takahashi, H. Kawanami, R. Ueda, Numerical investigation of the iterative phase-retrieval stagnation problem: territories of convergence objects and holes in their boundaries, J. Opt. Soc. Am. A 14 (1997) $3175-3187$.

[21] G. Yang, B. Dong, B. Gu, J. Zhuang, O. K. Ersoy, GerchbergcSaxton and Yang-Gu algorithms for phase retrieval in a nonunitary transform system: a comparison, Applied Optics 33 (1994) 209-218.

[22] J. Zhang, Y. Zhang, J. Lv, W. Liang, G. Situ, The design of diffractive optical elements with weighted serial iterative algorithm (in chinese), J. Optoelectronics 13 (2002) 1207-1210. doi:10.16136/j/joel.2002.12.001.

[23] A. V. Kuzmenko, Weighting iterative Fourier transform algorithm, Opt. Lett. 33 (2008) 1147-1149.

[24] H. H. Bauschke, P. L. Combettes, D. R. Luke, Phase retrieval, error reduction algorithm, and Fienup variants: a view from convex optimization, J. Opt. Soc. Am. A 19 (2002) 1334-1345.

[25] H. J. Levinson, Principles of Lithography, SPIE, 2005.

[26] F. Zhang, J. Zhu, W. Yue, J. Wang, Q. Song, G. Situ, F. Wyrowski, H. Huang, An approach to increase efficiency of DOE based pupil shaping technique for off-axis illumination in optical lithography, Opt. Express 23 (2015) 4482-4493. 